\title{
Information Technology Change Management in Networking
}

\author{
Manoj Mathew \\ IT Consultant \\ Bangalore, Karnataka, India
}

\begin{abstract}
IT Networks is always considered as complex to understand as it connects millions and millions of nodes across the globe and supposed to be active 24/7. New emerging technologies when combined with the existing systems can sometime bring instability and crash existing networks. This should be addressed immediately and the risks should be analysed before introducing changes into the corporate and global networks. In the global IT revolution, information technology change management is gaining more importance especially in the field of computer networks and applications. One such process followed in the corporate sector is Information Technology Change Management (IT ChM) which plays crucial role in the process of introducing and managing changes. This article focuses on the various issues faced and brings out the importance of IT infrastructure change management process.
\end{abstract}

Keywords- Change Management (ChM), Information Technology and Infrastructure Library (ITIL), Infrastructure, Request for Change (RfC).

\section{INTRODUCTION}

Information Technology infrastructure change management $(\mathrm{ChM})$ is a detailed process to control changes (add/remove/modify) on technology infrastructure, like applications, servers, network devices, Local Area Network (LAN), Wide Area Network (WAN) etc. This is applicable for all organizations irrespective of their size and spread of offices. All employees and clients across the globe are supposed to be connected to each other and managed by physical or virtual administrators. A robust Change Management process is mandatory to ensure the internal enterprise or Client ODC (Offshore Delivery Centre) IT infrastructures are stable and free from unforeseen incidents. It also gives control and visibility to senior management on their organizations technology infrastructure, and subsequently enables to sales and marketing team to forecast their delivery timelines with new clients.

The change management is helpful to the IT sector in various ways. Contractual obligation on technology service availability is one of the major nightmares for most of the CTO's, CIO's, and business managers. A stable change management process eases the life for managers by providing a nearly predictable technology infrastructure. This also helps the IT managers to efficiently forecast and streamline their resources. It enables delivery managers to plan their projects, as the change management reduces most of the surprise outages on their applications. A process oriented and reliable IT infrastructure is a dream for any clients to drive their products/business to success. Advanced planning of changes, enables operation/delivery managers to perform UAT (User Acceptance Test) and plan for their production activities. Finally, 99.9 \% SLA (Service Level Agreements) is possible only if you have a stable and reliable production environment.

A change management process to be invoked mainly of following situations:

i) A new application/software is getting into production or a new server is to be upgraded.

ii) Adding a new office network to an existing MPLS or Corporate Internet link.

iii) Launching a new high impact product and its Research and Development centre.

iv) Add/Remove/Modify any IT device configurations, like servers, routers, firewalls, switches, etc.

Typically, all IT changes which may impact the day-to-day activities of an organization must follow change management processes. The already existing system must be thoroughly monitored which aids in avoiding the cost leakages in the infrastructure management [1]. This is possible via NMS (Network Monitoring Systems) softwares in market and enables the technology management to monitor the infra from a remote location, and track/report/trigger changes almost real-time via NOC (Network Operation Centres). These tools also help as an input to the capacity planning teams for every component it monitors. For example, a threshold violation alert of above $70 \%$ usage on a network link is one of the key trigger to fund for that links upgrade. 


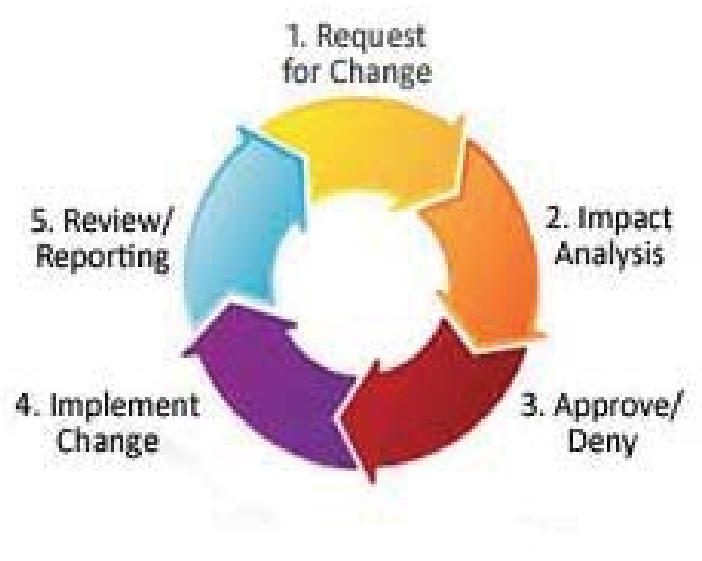

Figure 1. Change Management Process

The figure 1 shows the change management process that must be done in order to implement changes. The steps involved are:

i) Request for Change: Any change to an information technology resource is to be performed with a proper request for change through proper channel.

ii) Impact Analysis: When a change is subjected, the nature of the change must be analysed properly. Certain changes like adding a PC to the network happens regularly. Before approving or denying the changes, the impact analysis is done.

iii) Approve/Deny: All the changes must be conveyed through a proper Change Request Approval Process before it is implemented. Any request can have two possible outcomes; either it can be approved, if the changes does not bring worse effect, else it can be rejected. This change request approval process is a systematic procedure which is carried out by the Change Advisory Board (CAB), a set of IT service staff, who is appointed by the Director of the IT Services. These people will assemble and meet regularly for processing the Change Requests. The $\mathrm{CAB}$ has the rights to approve or deny the change requests subjected.

iv) Implement Change: Change Requests must receive $\mathrm{CAB}$ approval or authorization before proceeding to implement the changes. Once the $\mathrm{CAB}$ gives approval, the changes can be implemented within the scheduled time.

v) Review/Report: After implementing the changes, there must be a continuous monitoring on the implemented changes. Periodical reviews are made and reports are prepared accordingly, which signs as a continuous monitoring activity in order to ensure that the introduced change is working properly and does not affect the existing structure.

IT Services has the authority to repeal any unauthorised changes that cause, or suspected as causing, or they have the potential to cause interference to other users of the services.

The paper is organized as follows. Section II gives the importance of Change Management in Technology Infrastructure, Section III lists the various best practices followed by organizations on change management, Section IV gives a glimpse on the global standards on change management, Section V gives the change management tasks, Section VI highlights the hierarchy to be followed to implement change management, Section VII gives details on when to introduce change management, Section VIII throws insight on how to address emergency change management process, Section IX gives the change management in practice, Section $\mathrm{X}$ suggests some solution for effortless change management process, Section XI briefs out the various obstacles in introducing change management, Section XII gives the various tools available in the market for supporting change management process, Section XIII brings the advantages of change management and Section XIV gives the conclusion. 


\section{IMPORTANCE OF CHANGE MANAGEMENT IN TECHNOLOGY INFRASTRUCTURE}

Technology infrastructure is the backbone of any organization, and present business models cannot survive without a stable IT infrastructure. This is applicable for a small chain of medical shops to the latest Research and Development at NASA so technology in imperative for the growth of any company.

IT is not just software's, it includes networks, switches, routers, firewalls, servers, desktops, CCTV cameras and so on and so forth. To make them operational you need connectivity between each other, so there comes the terms LAN, MAN and WAN. In order to connect each LAN's we need WAN so in simple way LAN is a subset of WAN or several LAN's can be found in one WAN. WAN is a general term any company refers for their global networks, which connects to their offices or clients across the globe via several mediums, predominantly the undersea fibre cables link, or some cases our satellites used for mobile based services.

For example, once a new server is installed or an existing server is to be updated, after introducing the changes there needs a document change which must answer for 5W's and one ' $\mathrm{H}$ ' such as:

$\checkmark$ What is this for or business case?

$\checkmark \quad$ Why it is important?

$\checkmark$ When it is carried out?

$\checkmark$ Where it is done or location?

$\checkmark \quad$ Who is going to do it?

$\checkmark$ How it is implemented?

Once the answer is found for these issues, it must be appropriately updated on the corresponding documents such as the inventory list, network patch records, server recovery plans, maintenance records, and the system architectural documents etc is to be made for further follow ups.

These changes must be properly addressed. Change Management (ChM) is the key to sustain the global services via any of these devices deployed in an organization. When the company grows and spreads its office to multiple locations, they will lose the visibility to the infrastructure configurations as the number of devices are more for any human to remember. So, here comes the need of a process and that is called Change Management.

\section{VARIOUS BEST PRACTICES FOLLOWED BY ORGANIZATIONS ON CHANGE MANAGEMENT}

There are multiple best practices available in the industry on change management like ITIL, COBIT, PMP, and PRINCE, but an organization eventually decides what method suits them. A few best practices followed across the industry are:

i) ITIL - Information Technology and Infrastructure Library:

ITIL was initially developed by British Central Computer and Telecommunications Agency during 1980. The ITIL is considered as one of the best practice for change and configuration management which provides a general means for reaching the targets of Network Infrastructure Management [2]. ITIL calls the change management as a process and it takes inputs of Problem Management and Configuration Management. So, to make it simple, any changes in the IT infrastructure to be reviewed and approved by a group of experts in the respective areas and the name of the forum is $\mathrm{CAB}$ (Change Advisory Board), there are further divisions on it depends to the situations say, Emergency Change Advisory Board (ECAB), Regional Change Advisory Board $(\mathrm{RCAB})$ and Global Change Advisory Board (GCAB). It is applicable to the organization structure and decision can be made on what model suits the requirements. The term RfC in Change Management stands for Request for Change, which is the data what you will present to the CAB for their review and approval. The RfC can be a simple MS Excel template to sophisticated products like BMC's Remedy or HP's Service Desk.

\section{ii) Simple Ticketing System based ChM}

Here, the request will be attached to the home-grown or off the shelf ticketing tools and the approver/s will review and update their comments on the ticket. This is a cost effective solution for small firms; few disadvantages are a few poor tracking and lack of management reporting mechanisms.

iii) Automated Change Management Tools

In market, we can get several products which can be configured to automate configurations changes, CISCO's TACAS and CA's NMS are a few products in the market. 


\section{GLOBAL STANDARDS ON CHANGE MANAGEMENT}

The best standard to control the changes in a complex global IT infrastructure is ITIL v3 because the process there is so flexible, and can tune it as per our requirement. Following are some of the prerequisites for a successful change management process.

i) RfC template and a Change Review Script.

ii) A template for FSC (Forward Schedule of Changes), which is the proposed change schedules.

iii) A conference and web sharing medium like WebEx or Live Meeting account.

iv) List of your office locations and respective IT experts/approvers/reviewers list

v) List of your clients/vendors locations with their IT experts/approvers/reviewers list.

vi) Local and Global network diagrams.

vii) Details of the local and global data centres.

viii) List of your global servers and all critical devices in all locations.

When you get these ready, prepare a $\mathrm{CAB}$ with the representations from all locations and ensure they are aware of their responsibilities. An ideal CAB needs a CAB Manager, who will lead and control the general discussion and authorize/reject/postpone change requests. Change Requestor is another key member linked to every change/RFC, this person will readout the request in the forum/call and answer the queries from the $\mathrm{CAB}$ members.

\section{CHANGE MANAGEMENT TASKS}

The various tasks involved in change management are: i) Submit the RfC, ii) Review the submitted RFC, iii) $\mathrm{CAB}$ considers, analyse the changes and decide whether to approve or discard it, iv) If approved, then the changes can be implemented, and v) Post implementation review by CAB. The figure 2 shows a simple change process flow.

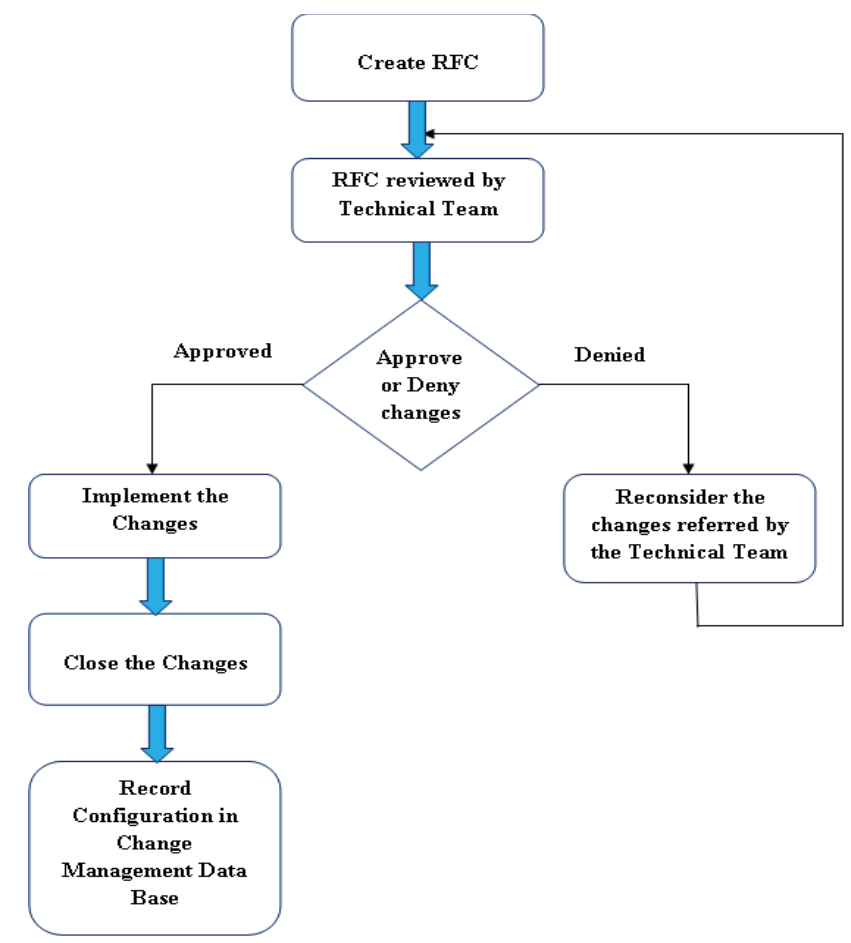

Figure 2. Simple Change Process Flow

VI. HIERARCHY TO BE FOLLOWED TO IMPLEMENT CHANGE MANAGEMENT

IT Change management should be managed by a forum called Change Advisory Board (CAB), where all the stakeholders are present and meets to review all changes on a predefined frequency (weekly/fortnightly/ monthly). Every CAB will have representations from all technical areas. The forum will be managed by a role 
by name CAB Manager, who will be the final approver for all changes. A "normal change" process follows the steps as follows:

i) A request for change is usually raised by a requestor that could be an engineer or a project manager.

ii) Updated RfC will be reviewed by respective technical team and inform the $\mathrm{CAB}$ manager to enlist on $\mathrm{CAB}$ agenda.

iii) The change requestor will present the change at $\mathrm{CAB}$ review and reads RfC script (high level explanation), and ensure the technical team (if need be) is aligned for answering queries.

iv) $\mathrm{CAB}$ then review the change to ensure it will not affect any other systems and approves/rejects the request.

\section{WHEN TO INTRODUCE CHANGE MANAGEMENT}

Change must be implemented before the next $\mathrm{CAB}$ meeting. Every change will be reviewed by immediate next $\mathrm{CAB}$ to record the results. All failed changes will be scrutinized before next attempt. Every time when a change is brought into consideration, obviously it takes time to identify the goodness of the change, get approval, and implement. So an organization must spend countable time in deciding the next move when a change is introduced. Often a question is placed on the time and how much time is wiser to spend in the change management process. The solution for this could depend on the organization and the number of changes across the company. Every change must be recorded and approved by CAB.

\section{ADDRESSING EMERGENCE CHANGE MANAGEMENT PROCESS}

Change types have been classified under 3 categories as: basic/normal, urgent, and standard. [3]. The basic/normal changes are associated with priority and are given considerations as per the priorities either low or medium or high or managed without CAB's review like service requests etc... The urgent change type must be addressed immediately and the standard change type is the usual one followed. Out of these three types, urgent or emergency changes need much more considerations. Emergency changes and Break Fixes are necessary for maintaining the projects workflow at some stages. Ideally such changes should be limited in overall count. Emergency changes are required when there is an unforeseen situation demand a change in environment but there will be approvals from top management or affected business group owners. Break fixes are needed when it's urgent and changes are required to fix an outage on production systems. Such changes may not be approved by anyone before implementation but the same will be reviewed in details at next $\mathrm{CAB}$ and ensure such changes are not repeated.

\section{CHANGE MANAGEMENT IN PRACTICE}

Current Change Management process carried in the industrial sector is an IT SLA of $99.9 \%$ is quiet normal on all businesses across industry. Failed changes and wrong updates on system configurations are major headaches for all organizations when there is not ChM process. A failed change is as simple as a business outage. More such failures results major risk to production and services to clients.

\section{SOLUTION FOR EFFORTLESS CHANGE MANAGEMENT}

Planned execution of a project is the best in any situation, irrespective of its size. So, any project results a change in the current system, it could be thru introduction or new methods or modifying the existing system.

i) Use a standard template for all change, which is called Request for Change template.

ii) Ensure you have a database of all your offices, functions and their respective key staffs.

iii) Any changes as part of IT projects or IT operations, to be strictly addressed and approved by the CAB (Change Advisory Board).

iv) Local IT support staff/managers to be responsible for the change and CAB Manager approves it.

v) All changes must be tested on preproduction environment and ensure an appropriate rollout plans are in place.

vi) Testing must be approved by end users group and the change window must be approved by the business owners and end users management. 
vii) UAT (User Acceptance Test) should be made mandatory for any changes.

viii) PIR (Post Implementation Review) is also an integral part of any changes, this ensures all changes are executed diligently and record any lessons learned during the change activity.

ix) A weekly/monthly change control report must reach senior management and ensure they review it thoroughly and strict actions are taken on all failed and unplanned changes.

x) Continues adherence to the policy enables the business run smoothly and provides stable infrastructure thus additional revenue for the organization.

\section{OBSTACLES IN INTRODUCING CHANGE MANAGEMENT}

In small and medium companies, senior management buy-in is the main obstacle for such a process implementation as mostly the response was that the ChM process increases workload of engineers and requires additional resources to manage it. This is the main misunderstanding and must to be addressed at top level if we need a successful ChM process.

\section{TOOLS IN MARKET FOR SUPPORTING CHANGE MANAGEMENT PROCESS}

There are few prominent tools available in market for supporting ChM process simple and easier. To name a few well know products:

- A simple MS Excel can do the work for tracking and use as a template for RFC.

- CA's Service Desk Management,

- HP Service Desk,

- $\quad$ BMC Remedy

\section{ADVANTAGES OF CHANGE MANAGEMENT}

Stability on production environment of any organization is sensitive for their business success and the ChM controls or minimizes surprise outages on IT environment and provides production stability. Following are a few benefits of change management:

i) Control on all IT infrastructure and production application developments/upgrades across the organization.

ii) Centralized reporting for executive management and enabler for growth/sales forecasts.

iii) Planned execution of changes and successful roll backs of failed changes.

iv) Proper and planned utilization of IT resources.

v) Avoid or eliminate production outages.

\section{CONCLUSION}

IT infrastructure change management is an important service in the technology management processes. Making any changes to the existing IT infrastructure or amendment is tedious as well as risky. When a change is introduced to one of the existing component, it will have a cascading effect on the interlinked components as well. Irrespective of the size of any organization, they might experience a large number of changes which needs to be controlled and carried out properly with planning; else it might collapse the existing application/network structure. Greater control over the infrastructure always reduces the complexity, cut down the time and saves the cost involved during transitions. Such IT infrastructure changes must be identified properly, analysed thoroughly and the relevant risks that production environment is expected to face must be addressed in prior, with clear planning and approval. In general, 70\% percentage of unplanned downtime is due to people errors and missing processes, which includes poor change management practices. Tested changes and approved processes increase the reliability as well as the availability of network that makes the company and its technology more predictable. If possible, the change management processes could be automated; it reduces time and cost of changes involved. Such automated changes might eliminate the manual process errors and the manpower involved.

\section{REFERENCES}

[1] Vaibhav Bhatia, Advanced Monitoring for IT Infrastructure, Infosys Lab Briefings, Vol. 9, No. 5, 2011.

[2] Antti Mattila, "Best practices for Network Infrastructure Management - A Case Study of IT Infrastructure Library (ITIL)"

[3] http://www.tewv.nhs.uk/Global/Policies\%20and\%20Procedures/IT/IT-0015-2\%20Information\%20Change\%20Control\%20Policy.pdf 